\title{
Effects of Titanium Impurity on the Crystallographic and Spin-rotation Transitions of FeS
}

\author{
Hyo-Duk Nam ${ }^{1}$ and Eng Chan Kim ${ }^{2 *}$ \\ ${ }^{1}$ Department of Electronics, Yeungnam University, Gyongsan 712-749, Korea \\ ${ }^{2}$ Department of Physics, Yeungnam University, Gyongsan 712-749, Korea
}

(Received 8 October 2010, Received in final form 17 February 2011, Accepted 17 February 2011)

\begin{abstract}
The effects of titanium ions on the crystallographic and spin-rotation transitions in iron sulfide have been examined by Mössbauer spectroscopy in the temperature range of 78 to $600 \mathrm{~K}$. It is noted that the titanium impurity of $\mathrm{Ti}_{0.02} \mathrm{Fe}_{0.98} \mathrm{~S}$ affects both the crystallographic and spin-rotation transitions of the iron sulfide. $2 \%$ impurity of $\mathrm{Ti}^{2+}$ in $\mathrm{FeS}$ causes the increase in the difference between the spin rotation and $\alpha$ transition temperature by as much as $10 \mathrm{~K}$ compared with that for FeS. Both $1 \mathrm{c}$ and $2 \mathrm{c}$ structures coexist in the range between the $\alpha$ transition temperature and approximately $26 \mathrm{~K}$, with a smaller hyperfine field corresponding to the $1 \mathrm{c}$ structure. The spin-rotation temperature for $\mathrm{Ti}_{0.02} \mathrm{Fe}_{0.98} \mathrm{~S}$ was measured to be $365 \mathrm{~K}$, which is $10 \mathrm{~K}$ lower than the $\alpha$ transition temperature. By the $2 \%$ impurity of $\mathrm{Ti}^{2+}$ in $\mathrm{FeS}$ the Néel temperature appreciably is not affected.
\end{abstract}

Keywords : titanium, FeS, spin rotation, crystallographic transition

\section{Introduction}

Interesting crystallographic and magnetic phase transitions appear in iron sulfide FeS.

It was reported that the crystallographic $\alpha$ transition took place at approximately $T_{\alpha} \approx 400 \mathrm{~K}$ in FeS [1]. According to the report, $\mathrm{FeS}$ exists in a NiAs structure above $T_{\alpha}$ and transforms to a hexagonal superstructure below this temperature. The length of the supercell c-axis is twice of the high-temperature phase. For that reason, the phases with the NiAs structure and the hexagonal superstructure will be called the $1 \mathrm{c}$ phase and $2 \mathrm{c}$ phase, respectively. Neutron-diffraction and magnetic susceptibility $[2,3]$ show that the $\mathrm{FeS}$ is antiferromagnetic with a Néel temperature of $\sim 600 \mathrm{~K}$. On comparison, for $\mathrm{Fe}_{0.996} \mathrm{~S}$ the spin-rotation transition (the Morin transition $T_{M}$ ) takes place at a $T_{M}$ temperature $31 \mathrm{~K}$ higher than $T_{\alpha}$.

In this paper we present our Mössbauer measurements for $\mathrm{Ti}_{0.02} \mathrm{Fe}_{0.98} \mathrm{~S}$ in an effort to determine the effects of $\mathrm{Ti}$ ions on the crystallographic and magnetic transitions of iron sulfides. As the impurity ions $\mathrm{Ti}^{2+}\left(3 \mathrm{~d}^{2}\right)$ ions have smaller number of $3 \mathrm{~d}$ electrons than that of $\mathrm{Fe}^{2+}\left(3 \mathrm{~d}^{6}\right)$ ions.

*Corresponding author: Tel: +82-53-810-2343

Fax: +82-53-810-4616, e-mail: eckim@ynu.ac.kr

\section{Experimental}

$\mathrm{Ti}_{0.02} \mathrm{Fe}_{0.98} \mathrm{~S}$ sample was prepared by direct reaction [4] of the elements. The starting materials were sulphur, iron, and titanium powders of $99.9999,99.995$, and $99.99 \%$ purity, respectively, accurately weighted by heating in an evacuated quartz tube. Mixtures of each elemental proportion sealed in vacuum quartz ampoules were first heated slowly to $600{ }^{\circ} \mathrm{C}$ to prevent explosion due to sudden expansion of sulphur gas, and then they were kept at this temperature for one day, followed by further heating to $1000{ }^{\circ} \mathrm{C}$ for seven days. After quenching down to room temperature in order to obtain a homogeneous material it was necessary to grind the sample and to press the powder into a pellet before the second annealing process at $1000{ }^{\circ} \mathrm{C}$ in an evacuated and sealed quartz ampoule for three days. A third firing similar to the second one was required to obtain the single-phase sample.

Extra precaution has been taken during the sealing process to avoid sulfur vaporization as shown in Fig. 1 [5]. The quartz tube with two narrow necks was first filled with the desired amount of sulfur and then covered by one layer of titanium and iron powder each. These two layers block light from the torch and reduce thermal energy transfer from the torch to the sulfur. Additionally, 


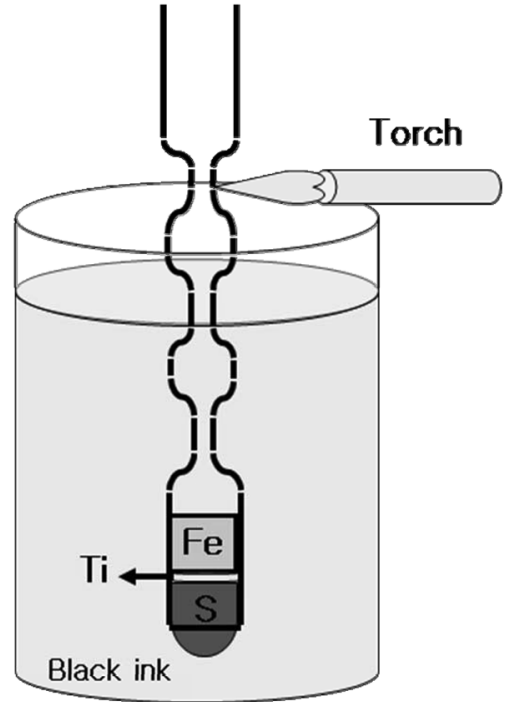

Fig. 1. Sealing Procedure.

the quartz tube was immersed into a liquid black ink bath to prevent sulfur vaporization due to the exposure to the light from the torch. Furthermore, the ink bath also can be used to cool the sample tube. During this sealing process the diffusion of sulfur vaporsinto the quartz tube is normally concerned. Since sulfur vapors in the sealed quartz ampoule would combine with iron and form solids on the tube wall during the initial heating at $450{ }^{\circ} \mathrm{C}$, at which temperature the diffusion is therefore negligible.

The sample was ${ }^{57} \mathrm{Fe}$ enriched to $5 \%$ of the metal atoms in the sample for Mössbauer measurements. Mössbauer spectra were recorded using a conventional electromechanical spectrometer manufactured by FAST Com Tec Ltd., which was set in a constant-acceleration mode with a 10 $\mathrm{mCi}{ }^{57} \mathrm{Co}$ source in a rhodium matrix. To produce a uniform thickness over the detection area of the Mössbauer absorber, each sample was mixed with boron nitride powder and clamped between two thin boron nitride plates.

The measurements below room temperature were made in a cryostat with the thermal stability of $0.25 \mathrm{~K}$ while those above room temperature were obtained in a furnace with the stability being $0.30 \mathrm{~K}$.

X-ray-diffraction patterns of $\mathrm{Ti}_{0.02} \mathrm{Fe}_{0.98} \mathrm{~S}$ at room temperature were obtained using a Rigaku diffractormeter with $\mathrm{Cu} K \alpha$ radiation.

\section{Results and Discussion}

$\mathrm{X}$-ray-diffraction patterns at room temperature showed that $\mathrm{Ti}_{0.02} \mathrm{Fe}_{0.98} \mathrm{~S}$ has the hexagonal superstructure. From which, the lattice parameters $a_{0}$ and $c_{0}$ were found to be

$$
a_{0}=0.9690 \pm 0.005 \AA \text { and } c_{0}=11.6892 \pm 0.05 \AA .
$$
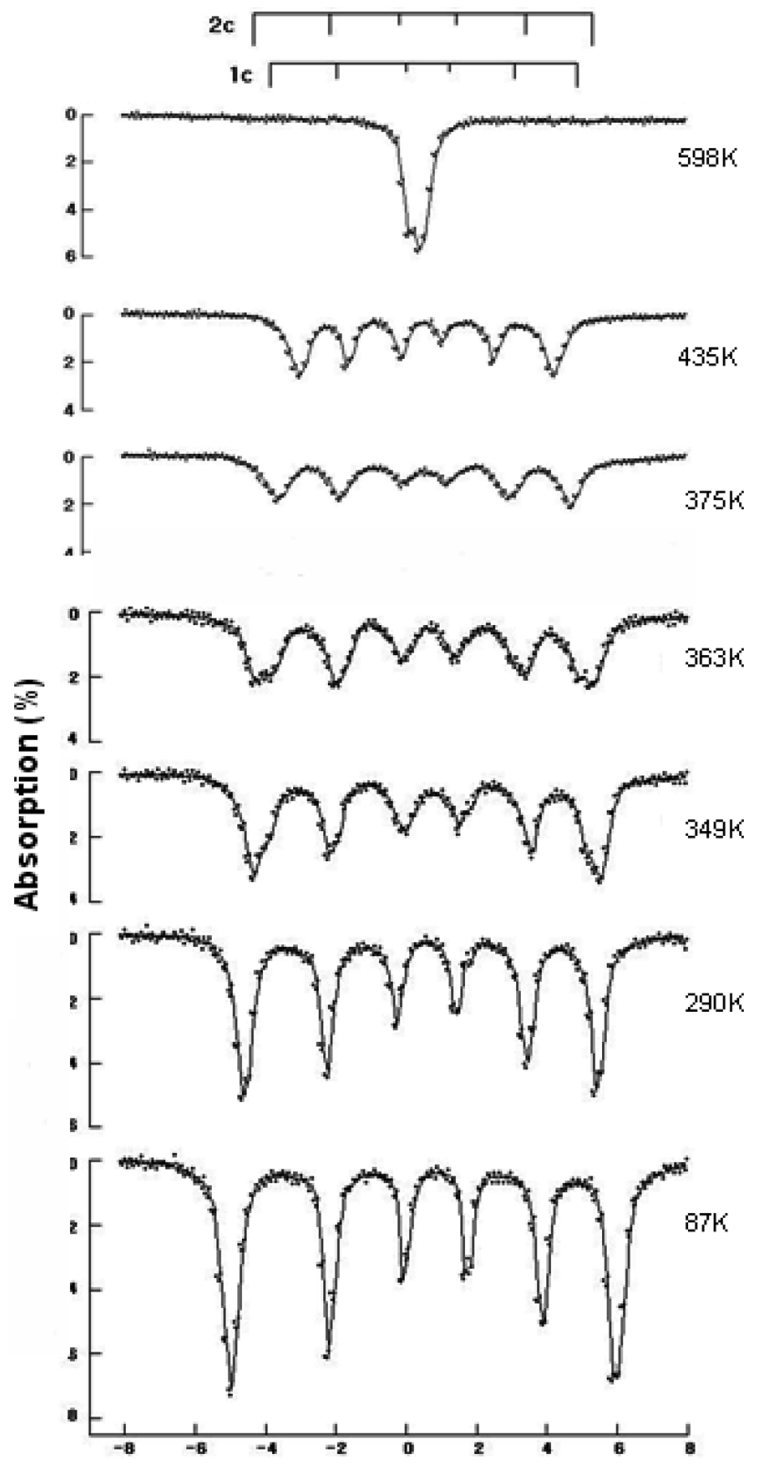

Fig. 2. Mössbauer spectra of $\mathrm{Ti}_{0.02} \mathrm{Fe}_{0.98} \mathrm{~S}$.

We have taken Mössbauer spectra of $\mathrm{Ti}_{0.02} \mathrm{Fe}_{0.98} \mathrm{~S}$ at various absorber temperatures ranging from 87 to $600 \mathrm{~K}$. They are shown in Fig. 2, which illustrates the temperature variations in the Mössbauer spectra for $\mathrm{Ti}_{0.02} \mathrm{Fe}_{0.98} \mathrm{~S}$. There are 6 Mössbauer lines and all peaks are very intense at both the high and low temperatures while 12 lines are present for the intermediate temperatures, suggesting the coexistence of $1 \mathrm{c}$ and $2 \mathrm{c}$ phases [6].

Figs. 3 and 4 reveal the temperature dependence of the quadrupole splitting $\Delta E_{Q}$ and the magnetic hyperfine field $H_{h f}$ for $\mathrm{Ti}_{0.02} \mathrm{Fe}_{0.98} \mathrm{~S}$, respectively. $\Delta E_{Q}$ was calculated from the positions of the Mössbauer absorption lines using the expression

$$
\Delta E_{Q}=\frac{1}{4}\left(V_{6}-V_{5}+V_{1}-V_{2}\right),
$$


Where $V_{i}$ represents the position of the $i$ th absorption line in $\mathrm{mm} / \mathrm{s}$.

Using a least-squares computer program, one set or two of the six Lorentzian lines were fitted to the Mössbauer spectra below the Néel temperature under the following restraints [7], which are valid if the quadrupole interaction is much weaker than the magnetic hyperfine interaction:

$$
\begin{aligned}
& E_{1}=\delta+\Delta E_{Q}+\frac{1}{2}\left(3 g_{1}+g_{0}\right) \mu_{N} H, \\
& E_{2}=\delta-\Delta E_{Q}+\frac{1}{2}\left(g_{1}+g_{0}\right) \mu_{N} H, \\
& E_{3}=\delta-\Delta E_{Q}+\frac{1}{2}\left(g_{0}-g_{1}\right) \mu_{N} H, \\
& E_{4}=\delta-\Delta E_{Q}-\frac{1}{2}\left(g_{0}-g_{1}\right) \mu_{N} H, \\
& E_{5}=\delta-\Delta E_{Q}-\frac{1}{2}\left(g_{1}+g_{0}\right) \mu_{N} H, \\
& E_{6}=\delta+\Delta E_{Q}-\frac{1}{2}\left(3 g_{1}+g_{0}\right) \mu_{N} H,
\end{aligned}
$$

where $E_{i}$ stands for the line position of the $i$ th absorption line in a six-line pattern. $\delta$ is the isomer shift. $\Delta E_{Q}$ represents the quadrupole shift:

$$
\Delta E_{Q}=\frac{1}{8} e^{2} q Q\left[e \cos ^{2} \theta-1+\eta \sin ^{2} \theta \cos (2 \phi)\right],
$$

where $\theta$ and $\phi$ are the angles, in polar coordinates, between the magnetic hyperfine field vector and the principal axes of the electric-field-gradient tensor. $g_{0}$ and $g_{1}$ represent the absolute values of $g$ factors for the ground and the first excited levels of the ${ }^{57} \mathrm{Fe}$ nucleus, respectively. $\mu N$ stands for the nuclear magneton and $H$ is the magnetic hyperfine field [5]. Therefore, from Eq. (3) we expect that $\Delta E_{Q}$ would change considerably at the spinrotation transition. It is apparent from Fig. 3 that Morin transition occurs at approximately $365 \mathrm{~K}$ which is $10 \mathrm{~K}$ lower than $T_{\alpha}$, the $\alpha$ transition temperature marking the upper end of the coexistence region of the $1 \mathrm{c}$ and $2 \mathrm{c}$

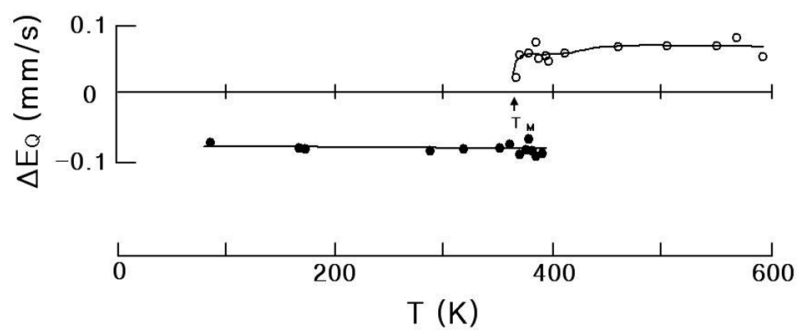

Fig. 3. Temperature dependence of the quadrupole splitting $\Delta E_{Q}$ for $\mathrm{Ti}_{0.02} \mathrm{Fe}_{0.98} \mathrm{~S}$.

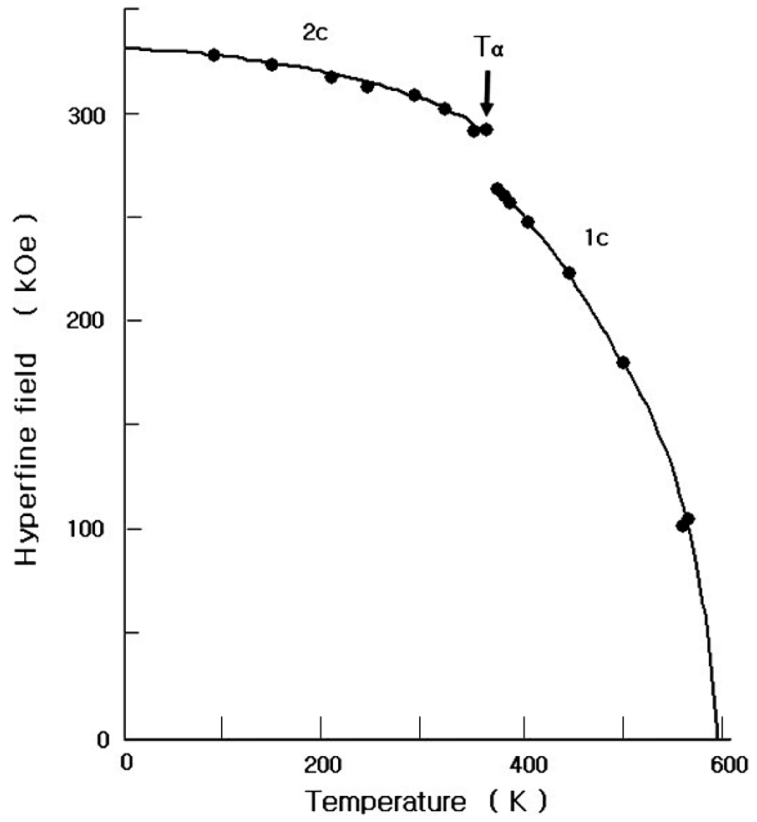

Fig. 4. Temperature dependence of the magnetic hyperfine field $H_{h f}$ for $\mathrm{Ti}_{0.02} \mathrm{Fe}_{0.98} \mathrm{~S}$.

phases as shown in Fig. 4. In Fig. 3 the quadrupole shift value for $\mathrm{Ti}_{0.02} \mathrm{Fe}_{0.98} \mathrm{~S}$ jumps abruptly at $365 \pm 1 \mathrm{~K}$ corresponding to the spin rotation from lying in the [001] plane above the transition to the pointing along the [001] direction below, which was observed in $\mathrm{FeS}[2,3]$. In addition there seems to be some outliers near $375 \mathrm{~K}$ related to the $\alpha$ transition in Fig. 4. Furthermore, the coexistence region of the two phases for $\mathrm{Ti}_{0.02} \mathrm{Fe}_{0.98} \mathrm{~S}$ is much narrower than $\mathrm{FeS}[6]$ as can be seen in Fig. 4. It is found that $2 \%$ titanium impurity of the metal atoms in FeS lead to rapid crystallographic transition in a narrow temperature region of about $26 \mathrm{~K}$, while the $\alpha$ transition in iron sulfide occurs over a wide temperature range of around $200 \mathrm{~K}$. Moreover, the spin-rotation temperature is increased by $10 \mathrm{~K}$ below $T_{\alpha}$ while $T_{M}$ for FeS is $24 \mathrm{~K}$ below $T_{\alpha}$.

The Néel temperature of $\mathrm{Ti}_{0.02} \mathrm{Fe}_{0.98} \mathrm{~S}$ was determined to be $598 \pm 2 \mathrm{~K}$, implying that the $2 \%$ titanium impurity in $\mathrm{FeS}$ does not affect the superexchange interactions in the 1c phase appreciably.

\section{Conclusion}

In conclusion, $\mathrm{Ti}^{2+}\left(3 \mathrm{~d}^{2}\right)$ impurity speeds up the crystallographic transition in a narrow temperature region of $26 \mathrm{~K}$ whereas there is a wide temperature range of approximately $200 \mathrm{~K}$ in iron sulfide [6]. In contrast, $\operatorname{Ti}^{2+}\left(3 \mathrm{~d}^{2}\right)$ impurity decreases the spin-rotation temperature by $10 \mathrm{~K}$ below $T_{\alpha}$ while $T_{M}$ for iron sulfide is $24 \mathrm{~K}$ below $T_{\alpha}$. 


\section{Ackowledgements}

This research was supported by the Yeungnam University research grants in 2008 .

\section{References}

[1] R. W. G. Wyckoff, Crystal Structures, Vol. 1, Interscience, New York, NY (1965) p. 24.

[2] J. T. Sparks, W. Mead, A. J. Kirschbaum, and W. Marshall, J. Appl. Phys. 31, 356 (1960).
[3] E. Hirahara and M. Murakami, J. Phys. Chem. Solids 7, 281 (1958).

[4] S. J. Kim, W. C. Kim, B. W. Lee, J. C. Sur, and C. S. Kim, J. Magn. Magn. Mater. 226, 518 (2001).

[5] K. S. Baek, K. Y. Park, H. J. Kim, and H. N. Ok, Phys. Rev. B 41, 9024 (1990).

[6] R. C. Thiel and C. B. van den Berg, Phys. Status Solidi 29, 837 (1968).

[7] C. S. Kim, I. B. Shim, M. Y. Ha, C. S. Kim, and J. Y. Park, J. Appl. Phys. 73, 5707 (1993). 